\title{
LOS CENTROS DE ESTUDIOS OLÍMPICOS COMO PARTE DE LA ESTRUCTURA DE LA ACADEMIA OLÍMPICA ESPAÑOLA: UNA INICIATIVA Y ALTERNATIVA PARA LA RENOVACIÓN Y ACTUALIZACIÓN DEL OLIMPISMO DENTRO DE LAS UNIVERSIDADES ESPAÑNOLAS
}

\author{
Carla Belén Gutiérrez Sánchez \\ $M^{\mathbf{a}}$ Eugenia Martínez Gorroño \\ Centro de Estudios Olímpicos de la Universidad Autónoma de Madrid
}

Fecha de recepción: Mayo de 2015

Fecha de aceptación: septiembre de 2015

http://dx.doi.org/10.15366/citius2015.8.2.003

\section{Resumen:}

La idea de llevar a cabo esta investigación nació con motivo de la participación en la convocatoria de becas de la Academia Olímpica Internacional (AOI) para el $22^{\circ}$ Seminario Internacional en Estudios Olímpicos para estudiantes de Posgrado. Tras la selección de los 35 beneficiarios, el seminario fue celebrado en septiembre de 2015, albergado en la sede de la IOA, en Olimpia, Grecia. Este trabajo será publicado por la IOA en inglés, uno de sus tres idiomas oficiales. La finalidad de este artículo es la difusión del proyecto mencionado entre la comunidad hispanohablante.

En ese estudio se abordó la creación, funcionamiento y evolución de los Centros de Estudios Olímpicos, instituciones dependientes de la Academia Olímpica Española integradas en Universidades de todo el territorio español. Su creación tiene como principales objetivos divulgar y expandir el Movimiento Olímpico bajo la rigurosidad del marco de la Educación Superior, marco en el que nació el Olimpismo Moderno. Las actividades desarrolladas por estas instituciones junto con un aumento significativo del número de Centros creados han contribuido y contribuyen a la difusión de la Educación y Valores del Movimiento Olímpico, sirviendo como ejemplo para su implantación en otros estados.

Palabras clave: Academia Olímpica Española, Academia Olímpica Internacional, Centro de Estudios Olímpicos, Comité Olímpico Español, Movimiento Olímpico. 
Title: OLYMPIC STUDIES CENTRES AS PART OF THE STRUCTURE OF THE SPANISH OLYMPIC ACADEMY: AN INITIATIVE AND ALTERNATIVE FOR RENEWAL AND UPDATING THE OLYMPISM BY SPANISH UNIVERSITIES.

\begin{abstract}
:
The premise for this research was born on account of the call for applications to participate in the 22nd International Seminar on Olympic Studies for Postgraduate Students, organised by the International Olympic Academy (IOA). Following selection of the 35 beneficiaries, the Seminar was held in September 2015 and hosted at the IOA headquarters, located in Olympia, Greece. This paper will be published by the IOA in English, one of its three official languages. The purpose of this paper is to disseminate the aforementioned study among the Spanish-speaking community.

In this study, the creation, operation and evolution of the Olympic Studies Centres are explored, as dependent institutions of the Spanish Olympic Academy, integrated into universities across the Spanish territory. The main objective of these Centres is to disseminate and expand the Olympic Movement, under the rigorous structure of the Higher Education framework in which the Modern Olympics was born. The activities of these institutions, accompanied by a significant increase in the number of Centres, have helped establish and contribute to the spread of Education and the values of the Olympic Movement, serving as an example for implementation in other countries.
\end{abstract}

Keywords: International Olympic Academy, Olympic Movement, Olympic Studies Centre, Spanish Olympic Academy, Spanish Olympic Committee

\title{
1. INTRODUCCIÓN
}

El propósito de esta investigación es dar a conocer la génesis y la estructura de los Centros de Estudios Olímpicos, entidades que como "delegaciones" de la Academia Olímpica Española actúan para cumplir los objetivos de la misma como difusores del Movimiento Olímpico, su filosofía, su ideario y su historia entre las comunidades universitarias españolas.

El sujeto de estudio de nuestra investigación, por tanto, son los Centros de Estudios Olímpicos lo cuales son una pieza más dentro del ámbito del Olimpismo, como se expondrá a lo largo de este trabajo.

\section{OBJETIVOS}

Tras el acercamiento preceptivo al objeto de estudio, el conocimiento adquirido como consecuencia del mismo nos suscitaba las siguientes inquietudes para el primer enfoque de nuestra investigación: 
1. ¿Qué son los Centros de Estudios Olímpicos?

2. ¿Para qué han sido creados?

3. ¿Qué tipo de trabajo han desarrollado y desarrollan estas instituciones?

Como consecuencia de las preguntas anteriores, planteamos los siguientes objetivos principales de nuestro proyecto:

1. Conocer la génesis y creación de la estructura de los Centros de Estudios Olímpicos dentro del funcionamiento de la AOE.

2. Analizar la estructura, actividades y evolución de estas delegaciones de la AOE.

3. Difundir la labor realizada por los Centros de Estudios Olímpicos y sus aportaciones al Movimiento Olímpico español e internacional.

\section{METODOLOGÍA}

Este trabajo supone una investigación de tipo cualitativo, pues responde a la afirmación de Rodríguez Gómez y García Jiménez que estimaron que la investigación cualitativa:

"Estudia la realidad en su contexto natural, tal y como sucede, intentando sacar sentido de, o interpretar los fenómenos de acuerdo con los significados que tienen para las personas implicadas". ${ }^{23}$

Por otro lado, se trata de un trabajo de investigación histórica cuya metodología se caracteriza como un abordaje sistémico de identificación, búsqueda y recogida de fuentes, seguido por la organización y evaluación de dichas fuentes y la estructuración de los datos que pueden proporcionar y que son relevantes en relación con el periodo objeto de estudio.

La labor de investigación histórica tiene muchas formas y puede abarcar muchos espacios y tiempos y por ello requiere de diferentes técnicas, herramientas e instrumentos para abordar el objeto de estudio, condicionada por las circunstancias del periodo elegido. El trabajo que aquí presentamos supone una investigación

${ }^{23}$ Rodríguez Gómez, G., García Jiménez, E. (1996). Metodología de la investigación cualitativa, p. 32. Granada: Ediciones Aljibe. 
histórica de un periodo y circunstancia de la Historia del Tiempo Presente del Olimpismo español, abordando un proceso temporal desde 1968 hasta el presente año.

La Historia del Tiempo Presente (HTP), ha sido definida por muchos autores y desde varias perspectivas. Así, por ejemplo Fazio Vengoa afirmaba que "la historia del tiempo presente es, ante todo, el estudio de un acontecimiento ocurrido en nuestra inmediatez", ${ }^{24}$ y Abdón Mateos, dice que "estudia preferentemente procesos históricos que, aunque sean recientes, están ya cerrados o para los que existe una mínima distancia cronológica", ${ }^{25}$ por lo que no se puede excluir del concepto de HTP a un pasado próximo o coetáneo a pesar de ser pasado. Mudrovcic, centrándose en la característica de la coetaneidad afirma que la HTP es "aquella historiografía que tiene por objeto acontecimientos o fenómenos sociales que constituyen recuerdos de al menos una de las tres generaciones que comparten un mismo presente histórico". ${ }^{26}$ Más recientemente Fazio Vengoa ha reflexionado sobre su condición de modernidad: "la historia del tiempo presente no es un término equivalente a contemporaneidad; designa, ante todo, aquel intervalo temporal en el cual se ha ido gestando la modernidad mundo, es decir, nuestro actual régimen de historicidad". ${ }^{27}$ Con ello quiere hacer alusión a su exclusividad en nuestro presente, añadiendo que: "sólo en este espacio de tiempo se ha asistido a un cambio tan radical en el curso de la historia". ${ }^{28}$

La Historia del Tiempo Presente muestra, unas diferencias significativas con la "historia tradicional". Por tanto, en este trabajo, fue necesario partir del concepto de HTP y de sus diferentes características para diseñar el proceso metodológico que debía llevarse a cabo en la investigación y que hemos realizado partiendo de los planteamientos metodológicos que desde hace años se siguen en el Departamento de Educación Física, Deporte y Motricidad Humana de la Universidad Autónoma de Madrid. Así, siguiendo las pautas publicadas por Martínez Gorroño, nuestro proceso metodológico ha tenido las siguientes etapas: diseño del cuestionario, realización de las entrevistas, transcripción de las entrevistas, tratamiento de la información oral, contraste y confirmación de la información obtenida en una segunda consulta (esta segunda entrevista que la citada

\footnotetext{
${ }^{24}$ Fazio Vengoa, H. (1998). La historia del tiempo presente: una historia en construcción p.52. Historia Crítica, 17, 47-57.

${ }^{25}$ Mateos López, A. (1998). Historia, Memoria, Tiempo Presente, p.1. Hispania Nova, (1). Recuperado de http://hispanianova.rediris.es/general/articulo/004/art004.htm.

${ }^{26}$ Mudrovcic, M. I. (2000). Algunas consideraciones epistemológicas para una "Historia del Presente". Hispania Nova, 1. Recuperado de http://hispanianova.rediris.es/general/articulo/013/art013.htm

${ }^{27}$ Fazio Vengoa, 2007, p. 195.

${ }^{28}$ Ibídem.
} 
autora denomina "de refrendo" o "de confirmación de datos" se realiza a fin de llevar a cabo posibles correcciones o confirmaciones de los datos obtenidos en la primera entrevista). Finalmente, procurando la validación de la entrevistas por parte del entrevistado, se procedió a la triangulación de los datos de los resultados del análisis de las otras fuentes documentales, escritas, gráficas, etc. procedentes de los diferentes archivos. ${ }^{29}$

Por tanto, teniendo en cuenta la circunstancia de la Historia del Tiempo Presente que supone este trabajo, una de las técnicas principales utilizadas ha sido la entrevista. En la actualidad, la historiografía especializada propone la entrevista como la técnica más idónea de acceso a las fuentes orales como fuentes primarias de la investigación, a fin de recabar su testimonio y rescatar sus recuerdos, para obtener una información de tipo cualitativo que permite conocer su opinión de una manera más extensa y personalizada.

Han sido realizadas tres entrevistas semiestructuradas de final abierto ${ }^{30}$ a dos fuentes primarias protagonistas de los hechos objeto de estudio: El Presidente de la Academia Olímpica Española, Conrado Durántez Corral, y $\mathrm{M}^{\mathrm{a}}$ Eugenia Martínez Gorroño, Miembro de la Academia Olímpica Española y Directora del Centro de Estudios Olímpicos de la Universidad Autónoma de Madrid (UAM), creado a partir de su iniciativa.

\section{LA ACADEMIA OLÍMPICA ESPAÑOLA: PUNTO DE PARTIDA DE LOS CENTROS DE ESTUDIOS OLÍMPICOS}

El 25 de noviembre de 1968, tras el visto bueno del Presidente del Comité Olímpico Español (COE), Juan Antonio Samaranch Torelló, ${ }^{31}$ se fundó la Academia Olímpica Española (AOE). El gran impulsor y Presidente de la misma desde aquel día hasta la actualidad es D. Conrado Durántez Corral. Él había sido el enviado por el COE como representante de España en la primera sesión de la Academia Olímpica Internacional (AOI), celebrada en Olimpia, en junio de 1961. ${ }^{32}$

\footnotetext{
${ }^{29}$ Martínez Gorroño, M.E. (1996). Metodología y fuentes en el estudio del exilio español en Colombia a consecuencia de la guerra civil 1936-1939. En J.M Trujillano Sánchez y P. Díaz Sánchez (Ed.), Actas y Jornadas Historia y Fuentes orales-testimonios orales y escritos, (pp. 253-258). Ávila, España: Fundación Cultural Santa Teresa.

${ }^{30}$ Hammer, D. y Wildavsky, A. (1990). La entrevista semi-estructurada de final abierto. Aproximación a una guía operativa. Historia y Fuente Oral, 4, 23-61.

${ }^{31}$ Juan Antonio Samaranch Torelló fue Presidente del Comité Olímpico Español desde 1967 hasta 1970 y del Comité Olímpico Internacional desde 1980 hasta 2001. En http://www.elpais.com/especial/juanantonio-samaranch/vida.html

${ }^{32}$ Academia Olímpica Española. (1990). Academia Olímpica Española XXII Sesión: XX Aniversario. Madrid: Comité Olímpico Español.
} 
En un primer momento, la AOE no se constituyó dentro de la estructura del COE. Si bien contó con su apoyo desde el inicio, no se había aún gestado la idea de una institución semejante dentro de las estructuras de los comités nacionales (CON). El COE se encargaba de enviar a los representantes en la sesión anual de la AOI desde su primera celebración en 1961, apoyando la idea surgida dentro del Movimiento Olímpico de contar dentro de su estructura con una institución encargada de velar por el ideario olímpico y transmitir a las nuevas generaciones su ideario y su historia. De esta forma, se creó bajo el nombre de Centro de Estudios Olímpicos dentro del Instituto Nacional de Educación Física (INEF) de Madrid, institución que impartía formación superior en el área de las CCAFyD, que en aquellos años estaba bajo la dirección de José $\mathrm{M}^{\mathrm{a}}$ Cagigal.

Posteriormente, en 1973 pasó a denominarse de forma definitiva Academia Olímpica Española. Más tarde nuevas Academias Olímpicas Nacionales fueron surgiendo como la de EE.UU (1976), China Taipei (1978), Japón (1978), etc. ${ }^{33} \mathrm{Si}$ bien la creación del Centro de Estudios Olímpicos español, fue una aportación pionera y definitiva para el olimpismo español, el olimpismo internacional también lo estimó como una contribución de singular importancia. Así, tras los primeros años de funcionamiento del Centro de Estudios Olímpicos en el INEF de Madrid, en 1971, durante la celebración del X Aniversario de la AOI, el COE y el Presidente de la AOE, Conrado Durántez Corral, fueron designados Colaboradores Oficiales de la AOI. Durántez fue además nombrado como nuevo miembro de la AOI. ${ }^{34}$

En 1987, debido al nuevo organigrama con el que se configuró el COE, la AOE pasó a formar parte de su estructura con la creación de una Comisión para la Academia. ${ }^{35}$

En 1964 Cagigal asistió a la Sesión de la AOI a propuesta de Durántez, quien había asistido a todas las sesiones celebradas por la misma hasta esa fecha. Cagigal volvió entusiasmado de Olimpia, convencido en forma definitiva, de las sugerencias que venía haciéndole Durántez Corral, relativas a que el ideario olímpico era una filosofía cargada de valores positivos de gran interés para los estudiantes del INEF de Madrid, que serían los futuros especialistas españoles en el área de las Ciencias de la Actividad Física y el Deporte. Aquella circunstancias fue el inicio de que

\footnotetext{
${ }^{33}$ Academia Olímpica Internacional. Recuperado de http://www.ioa.org.gr/?lang=en\&item $=84 \&$ view $=1 \&$ art_id $=246$

${ }^{34}$ Martínez Gorroño, M. E. (2015). Conrado Durántez Corral: La Academia Olímpica Española y el olimpismo. Materiales para la Historia del Deporte, [S.1.], 158-181. Universidad Pablo de Olavide de Sevilla. ISSN 1887-9586. Disponible en:

<http://www.upo.es/revistas/index.php/materiales_historia_deporte/article/view/1230/985>.

${ }^{35}$ Ibídem.
} 
acordase junto con Conrado Durántez la creación de una institución a nivel nacional con los mismos objetivos que la AOI, que fuese delegada de la misma, ${ }^{36}$ y que, como dijimos, empezó a funcionar dentro del INEF de Madrid en 1968.

Entre las funciones y labores de la AOE se estableció la celebración de una sesión anual, de forma análoga a la AOI. La primera Sesión de la Academia Olímpica Española se celebró en 1969, en el INEF de Madrid, que además también se llevó a cabo con el objetivo de preparar a los estudiantes del INEF que serían seleccionados para participar en la Sesión para Jóvenes Participantes de la Academia Olímpica Internacional. ${ }^{37} \mathrm{~A}$ partir de aquella fecha, ambas tareas han sido funciones que la AOE ha venido repitiendo anualmente: la celebración de la Sesión, a manera de curso intensivo con conferencias de tema olímpico a cargo de especialistas, y la selección, entre sus participantes, de los candidatos representantes de España para la sesión anual de la AOI que celebra en Olimpia.

La creación a partir de 1980 de nuevos Institutos Superiores de Educación Física hizo que la AOE se plantease extender la celebración de las sesiones de la Academia a las sedes de los nuevos INEFs, para así cumplir su función de difusora del ideario olímpico y el Olimpismo entre las nuevas comunidades universitarias. Se celebraron sesiones en los Institutos de Barcelona (1984), Lérida (1987) y Granada (1989). ${ }^{38}$ La AOE consideró importante esta ampliación por su labor divulgativa y por el mecanismo de selección de candidatos para asistir a la sesión anual de la AOI, que al producirse en el contexto universitario y variar de sede, la AOE lo valoró como una oportunidad de aumentar la arbitrariedad en el proceso de selección, haciendo de este un proceso más justo. ${ }^{39}$

En 1988 la sesión de la AOE se celebró en el INEF de Lérida de forma simultánea al encuentro nacional denominado INTERINEFs (encuentro anual de los Institutos Nacionales de Educación Física de diferentes regiones de España. En aquel año eran once centros). Por primera vez se inauguró la Exposición Olímpica en la que se mostraron todos los carteles de los Juegos Olímpicos, la Filatelia y bibliografía olímpicas, así como se proyectaron filmaciones especializadas en la

\footnotetext{
${ }^{36}$ Durántez Corral, C. (2013). Entrevista oral celebrada en el COE, el 18 de abril de 2013. Disponible en el Centro de Estudios Olímpicos de la Universidad Autónoma de Madrid.

37 Cagigal J. M. (1973) Centro de Estudios Olímpico, p. 5. Madrid. Archivo del Consejo Superior de Deportes. Obra citada en Tarín Mariscal, M. (2013). Historia de la génesis de la Academia Olímpica Española. Contexto, herencia y consecuencias de su creación. Citius, Altius Fortius. Humanismo, Sociedad y Deporte: Investigaciones y ensayos, 6(2), 39-59. Centro de Estudios Olímpicos de la Universidad Autónoma de Madrid. http://cdeporte.rediris.es/revcaf/CitiusAltiusFortius.html 38 AOE. (2009). Academia Olímpica Española. 1968-2008. XL Aniversario. Madrid: COE.

${ }^{39}$ AOE, op. cit., 1990.
} 
temática olímpica. ${ }^{40}$ Posteriormente las sucesivas sesiones anuales de la AOE han sido celebradas simultáneamente con otras actividades: exposición de carteles olímpicos, mesas redondas con deportistas olímpicos, vídeos, etc. Para ello la AOE, fue creando un fondo de Exposiciones que fue poniendo a disposición de los diferentes CEOs de las universidades para que pudieran acompañar y crear un clima adecuado para la celebración de la sesión anual. Estas exposiciones, que hoy forman parte de los fondos de la AOE, han sido creadas por iniciativa de Conrado Durántez. Están conformadas con documentación escrita y gráfica que han sido fuentes primarias de los trabajos de investigación histórica llevados a cabo por Conrado Durántez en torno a la vida de Pierre de Coubertin y la génesis del Olimpismo. Han sido recabadas a partir de los archivos personales y familiares de la familia de Pierre de Coubertin y de los fondos del COI en Lausana. Una de ellas bajo el nombre de "Pierre de Coubertin. El Humanista olímpico" es única en su género, suponiendo la exposición más importante de material directo de los archivos familiares de Pierre de Coubertin, y que es un seguimiento único de su vida y su obra.

A partir de la década de los ochenta se puede afirmar que, la AOE se había extendido en el ámbito universitario, lo cual explica la creación de las siguientes instituciones: los Centros de Estudios Olímpicos.

\section{CENTROS DE ESTUDIOS OLÍMPICOS}

\subsection{Origen y misión de los Centros de Estudios Olímpicos}

Los Centros de Estudios Olímpicos (CEOs) se han ido creando como delegaciones de la AOE en las Universidades para cumplir los objetivos de la misma dentro de sus respectivas comunidades universitarias. ${ }^{41}$ Para poder iniciar aquel proceso, Conrado Durántez, en su condición de jurista, elaboró y redactó un convenio que sería el texto común para la formalización de dicha creación, que debía ser suscrito por el Comité Olímpico Español y las diferentes universidades que decidieran crear dentro de su estructura un Centro de Estudios Olímpicos. ${ }^{42}$

En sus escritos y conferencias Conrado Durántez siempre recuerda que el Movimiento Olímpico no surgió en un gimnasio, ni en un campo de deportes, sino

\footnotetext{
40 Ibídem.

${ }^{41}$ Martínez Gorroño, M. E. (2015). Conrado Durántez Corral: La Academia Olímpica Española y el olimpismo. Materiales para la Historia del Deporte, [S.1.], 158-181. Universidad Pablo de Olavide de Sevilla. ISSN 1887-9586. Disponible en:

<http://www.upo.es/revistas/index.php/materiales_historia_deporte/article/view/1230/985>.

42 COE. (2007a). 1er Congreso Nacional de Centros de Estudios Olímpicos. Universidad de Granada, 22 y 23 de marzo de 2007. Madrid: COE.
} 
en una Universidad: la Sorbona de París. ${ }^{43}$ En base a este deseo de devolver el Movimiento Olímpico a la universidad, como primer paso, se acordó crear el Centro de Estudios Olímpicos de la Universidad de Barcelona en su Campus de Bellaterra. Aquel fue el primero de una serie que aún se sigue incrementando. Se firmó el convenio el 28 de Junio de 1989 utilizando una fórmula similar a la utilizada en el año 1968 para la creación del Centro de Estudios Olímpicos en el Instituto Nacional de Educación Física de Madrid.

Sobre cómo surgió aquella idea y la razón por la que se inició aquel esfuerzo que hoy supone una verdadera estructura que se extiende por todo el territorio español, Conrado Durántez nos decía en una entrevista que con él mantuvimos en su despacho de la Academia Olímpica en Madrid:

"La Academia tiene una dimensión genérica en la sociedad, desde la escuela al adulto, pero el lugar suyo es la universidad, que es donde nació el Movimiento Olímpico, nació en la Sorbona, por Pierre de Coubertin en 1894. La universidad es el lugar donde un señor, con el estatus mental de un universitario, puede comprender la llamada humanista del Olimpismo, por eso creo que es el lugar adecuado, para que los Centros fuesen los difusores de esos principios en sus territorios. El ambiente adecuado de lo que es la difusión olímpica es la universidad." 44

La AOE ha venido manteniendo y mantiene, a partir de la estructura de los CEOs, una vinculación directa con la universidad desde su origen. Así ha ido celebrando en los campus universitarios su anual Sesión de la Academia Olímpica Española: de las 45 sesiones celebradas hasta la fecha, 44 lo han hecho en sedes universitarias. Así pues, la relación entre Academia y el ámbito universitario es anterior a la creación de los CEOs, lo cual apoya las palabras anteriores de Durántez. Por otro lado, y contemplando también otro rol que la universidad puede cumplir a favor del Olimpismo, Durántez escribió que también se espera que presten especial atención al campo de la investigación de la historia, ética y pedagogía del olimpismo, al máximo nivel académico como corresponde al ámbito universitario. ${ }^{45}$

Los CEOs han sido creados con la finalidad de expandir la labor de la AOE, ampliando el ámbito de actuación de la misma a través de la realización de actividades de difusión del Movimiento Olímpico en las Universidades donde estén situados y siempre con la colaboración, apoyo y actuación conjunta de la AOE. ${ }^{46}$

${ }^{43}$ COE, op. cit., 2007a.

${ }^{44}$ Durántez Corral, op. cit., 2013

${ }^{45}$ COE, op. cit., 2007a.

${ }^{46}$ Página oficial del COE, sección de la AOE. Consultado en

Citius, Altius, Fortius Volumen 8 ne 2 Noviembre, 2015. ISNH. 2340-9886. http://dx.doi.org/10.15366/eitius2015.8.2 
Los objetivos generales que la AOE ha cumplido y viene cumpliendo a través de los CEOs son los siguientes: ${ }^{47}$

- Llegar a un mayor número de personas, con la presencia en diferentes regiones del país, de centros especializados en Olimpismo.

- Acercar el Movimiento Olímpico y por ende la pedagogía Olímpica, a los centros de educación superior.

- Abrir una puerta, a la posibilidad de realizar proyectos de investigación de temática olímpica, dentro de las propias Universidades.

- Aumentar en definitiva los activos de la Academia.

\subsection{Funcionamiento y actividades de los CEOs}

En relación a los procedimientos seguidos y acuerdos tomados para la creación de un CEO, Conrado Durántez indicaba que: ${ }^{48}$

"El CEO en cuestión se crea por la vía de un Convenio suscrito por el Presidente del COE, el Rector de la Universidad respectiva y el Presidente de la AOE. El COE$A O E$ cede un fondo bibliográfico de temática olímpica al CEO y ambas partes contratantes se comprometen a organizar ciclos de conferencias, seminarios o cursos de difusión olímpica, colaborando proporcionalmente en los costes."

El proceso de apertura de un CEO es el siguiente: ${ }^{49,50}$

- Convenio firmado por las tres instituciones: AOE, COE y Universidad.

- Designación de un Director o Directora del CEO entre los integrantes de la Universidad en la que se crea.

- Nombramiento de una Comisión de Seguimiento de 6 miembros, integrada por 3 miembros de la AOE y tres propuestos por la Universidad.

http://www.coe.es/2012/HomeOlimpismo.nsf/FHomeOlimpismoN3?ReadForm\&Nivel3=Academia\% $20 \mathrm{Ol} \% \mathrm{C} 3 \%$ ADmpica

47 Archivo de la AOE y página web oficial de la AOE.

${ }^{48}$ Durántez Corral, op. cit., 2013.

${ }^{49}$ COE, op. cit., 2007a.

${ }^{50}$ Convenio de la Universidad Autónoma de Madrid (UAM). Archivo del CEO de la UAM.

Citius, Altius, Fortius Volumen 8 no 2 Noviembre, 2015. ISHN. 2340-9886.

http://dx.doi.org/10.15366/eitius2015.8.2 
- Entrega de una dotación de bibliografía olímpica al CEO por parte del COEAOE.

- Compromiso de programación de actividades de difusión e investigación del olimpismo.

Durante la actual etapa de la Presidencia del COE que corresponde a Alejandro Blanco Bravo, se ha manifestado un interés e impulso práctico para ampliar el número de CEOs y apoyar su funcionamiento. Analizando los datos sobre la apertura de nuevos CEOs se puede observar una evolución positiva en este aspecto, creándose 7 centros en la década de los años 90 (1989-1999), 17 en los 10 años siguientes (2000-2009) y 11 en los últimos 5 años (2010-2014).

En la actualidad, las Sesiones de la AOE se celebran anualmente en colaboración con el CEO de una universidad diferente, rotando cada año de universidad y de región. Dado que en el marco de las Sesiones de la AOE se realiza la elección de estudiantes que acuden a la Sesión para Jóvenes Participantes de la $A O I$, al CEO implicado en cada sesión le corresponde la función compartida (con la AOE) de realizar dicha selección, bajo los criterios generales establecidos por la AOE que se aplican cada año.

Según hemos podido comprobar a partir de los testimonios recogidos en las entrevistas contrastados con los datos que nos han ofrecido la revisión de las Actas de las diferentes sesiones celebradas hasta la fecha, las Sesiones de la AOE suponen unos cursos que suelen incluir un ciclo de conferencias a cargo de miembros de la AOE, profesorado universitario especializado en la temática olímpica y deportistas $\mathrm{u}$ otras personalidades relevantes y vinculadas al Olimpismo.

Una función más de los CEOs es la de publicar un libro de Actas de la Sesión Anual de la AOE, siendo responsable de su edición el CEO de la universidad donde se ha celebrado la misma. Dichas actas han ido ampliando la bibliografía especializada de temática olímpica publicada en español, que una vez editadas, cada año, se remiten por correo postal a todos los CEOs. Estas actas han ido incrementando cada año el fondo bibliográfico de sus bibliotecas que fue iniciado con la cesión del fondo bibliográfico de temática olímpica que fue cedido por el $\mathrm{COE}$ como consecuencia de la firma del convenio que supuso su creación.

En materia de publicaciones, además de las Actas que acabamos de mencionar, existen otras publicaciones impulsadas por los CEOs. Un claro ejemplo es la publicación de la revista de investigación Citius, Altius, Fortius. Humanismo, sociedad y deporte: Investigaciones y ensayos, iniciativa del Centro de Estudios Olímpicos de la Universidad Autónoma de Madrid. Esta revista comenzó a editarse 
en el año 2008, versión en papel, semestralmente y bajo la dirección de Conrado Durántez Corral y la $\mathrm{M}^{\mathrm{a}}$ Eugenia Martínez Gorroño, Profesora de la Universidad Autónoma y Directora del Centro de Estudios Olímpicos de la UAM, con el fin de difundir en español trabajos de investigación de calidad que sobre el Movimiento Olímpico y el Olimpismo se están produciendo a nivel español e internacional y ponerlos a disposición de la comunidad Hispanohablante. ${ }^{51}$ En su intervención en el Congreso Internacional de Historia del Olimpismo "Conrado Durántez" celebrado en Sevilla en Octubre de 2014, Martínez Gorroño hacía referencia a la citada publicación:

Tabla 1. Número de Centros de Estudios Olímpicos creados desde 1989 a 2014

\begin{tabular}{|c|c|c|c|}
\hline Años & 1989-1999 & 2000-2009 & 2010- 2014* \\
\hline 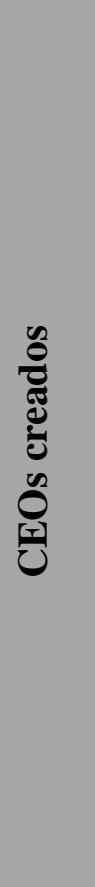 & $\begin{array}{l}\text { Universidad Autónoma } \\
\text { de Barcelona } \\
\text { Universidad de A } \\
\text { Coruña } \\
\text { Universidad de } \\
\text { Granada } \\
\text { Universidad de Cáceres } \\
\text { Universidad de León } \\
\text { Universidad de } \\
\text { Almería } \\
\text { Universidad del País } \\
\text { Vasco }\end{array}$ & $\begin{array}{l}\text { Universidad Católica San Antonio de } \\
\text { Murcia } \\
\text { Universidad de Santiago de } \\
\text { Compostela } \\
\text { Universidad de Pablo de Olavide de } \\
\text { Sevilla } \\
\text { Universidad de Córdoba } \\
\text { Universidad de Alicante } \\
\text { Universidad Rey Juan Carlos de } \\
\text { Madrid } \\
\text { Universidad de Valladolid en Segovia } \\
\text { Universidad Autónoma de Madrid } \\
\text { Universidad de Valencia } \\
\text { Universidad Europea Miguel de } \\
\text { Cervantes de Valladolid } \\
\text { Universidad de Cádiz, } \\
\text { Universidad Alfonso X El Sabio } \\
\text { Ayuntamiento de Noja } \\
\text { Universidad de La Rioja } \\
\text { Universidad de Jaén } \\
\text { Universidad de Vigo } \\
\text { Universidad de Oviedo }\end{array}$ & $\begin{array}{l}\text { Universidad de Las } \\
\text { Palmas de Gran } \\
\text { Universidad de } \\
\text { Valladolid en Soria } \\
\text { Fundación Barcelona } \\
\text { Olímpica } \\
\text { Ayuntamiento de } \\
\text { Castellón } \\
\text { Universidad de Navarra } \\
\text { Universidad Europea de } \\
\text { Madrid } \\
\text { Universidad de Alcalá de } \\
\text { Henares } \\
\text { Universidad de Cantabria } \\
\text { Ayuntamiento de La } \\
\text { Nucía } \\
\text { Universitat de Girona } \\
\text { Universidad de Castilla } \\
\text { la Mancha }\end{array}$ \\
\hline Total & 7 & 17 & 11 \\
\hline
\end{tabular}

${ }^{51}$ Martínez Gorroño, op. cit., 2015, pp.164-165. 
"Con ello estamos contribuyendo, no sólo a la difusión del olimpismo entre nuestras comunidades universitarias hispanohablantes, sino a ofrecer un cauce de publicación reputada a los investigadores que sobre el olimpismo decidan encauzar sus esfuerzos en líneas de investigación relacionadas con el Movimiento Olímpico, aceptando manuscritos originales de Investigaciones sobre recuperación histórica y proyección social y humanística del Olimpismo, Movimiento Olímpico y el deporte. Los manuscritos son sometidos a doble evaluación anónima externa, como es preceptivo en este tipo de publicaciones de rigor que como criterio esencial se exige en las publicaciones internacionales. En estos momentos nos encontramos en un proceso para su digitalización en la dirección:

cdeporte.rediris.es/revcaf/CitiusAltiusFortius.html". ${ }^{52}$

Otro CEO especialmente activo es el de la Universidad del País Vasco que desde 1999 ha venido organizando anualmente la Semana Olímpica de Bilbao. Dicha evento consta, entre otros actos, de ciclos de conferencias programadas en base a tres ejes de actuación: ${ }^{53}$

- Nuevos Publicadores

- Investigadores del ámbito estatal

- Deporte Vasco

En 2010 este CEO publicó la obra De Re Olímpica a la que ha seguido un segunda parte publicada en el 2013, en la que se recogen las conferencias impartidas en cada una de las ediciones de la Semana Olímpica.

\subsection{Avances, progresos e innovaciones en la estructura de los CEOs.}

Como consecuencia del incremento del número de CEOs, se creyó conveniente en el 2007 la celebración en la Universidad de Granada del I Congreso Nacional de CEOs. Se trataba de realizar una puesta en común del trabajo llevado a cabo y poder valorar de forma conjunta por todos los miembros los logros obtenidos y detectar, tanto las posibles dificultades particulares de cada centro, como las comunes a todos. Asistieron cada uno de los Directores y Directoras de los centros existentes en aquel momento, quienes expusieron la memoria de actividades de cada uno de los CEOs.

\footnotetext{
${ }^{52}$ Ibídem.

${ }^{53}$ COE, op cit., 2007, p. 86.
} 
A partir de esa fecha cada dos años se celebra un nuevo encuentro entre CEOs, el último de ellos en el 2013, el IV Congreso Nacional de CEOs en Noja (Santander).

Una de las novedades dentro de los integrantes de entidades registradas como CEOs, en los últimos años se ha producido la inclusión de entidades no universitarias, tales como Ayuntamientos y Fundaciones. Se trata de entidades, que por su recorrido o interés han sido valoradas positivamente por el COE y la AOE abriendo nuevas vías de difusión del Movimiento Olímpico. Dichos CEOs en la actualidad son cuatro: Ayuntamiento de Noja, Fundación Barcelona Olímpica, Ayuntamiento de Castellón y Ayuntamiento de La Nucía.

El objetivo que ha motivado esta innovación ha sido ampliar las posibilidades de expansión y divulgación del olimpismo y sus valores por todo el territorio español. ${ }^{54}$

Si bien estimamos expuestas las motivaciones que dentro de la $\mathrm{AOE}$ suscitaron el inicio y la creación de la estructura de los Centros de Estudios Olímpicos dentro de las universidades, también hemos intentado recabar, a partir de las fuentes orales, la motivación que puede estar detrás del impulso y aceptación de las universidades para su creación. Sobre ello nos respondía la profesora $\mathrm{M}^{\mathrm{a}}$ Eugenia Martínez Gorroño, Directora del CEO de la Universidad Autónoma de Madrid, e impulsora dentro de su estructura de la firma del citado convenio que fue suscrito para su creación:

"Teniendo en cuenta que la Universidad Autónoma de Madrid imparte el grado universitario de Ciencias de la Actividad Física y el Deporte y que además está inmerso dentro de la Facultad de Formación de Profesorado y Educación, nos parecía idóneo y de gran interés para nuestro alumnado la creación de un Centro de Estudios Olímpicos, que sirviera para acercar a nuestra comunidad universitaria especialista al Movimiento Olímpico y al ideario del olimpismo. Una Facultad de Formación de Profesorado y Educación tiene muchas sinergias con el ideario olímpico. Por otra parte, esto suponía que nuestro alumnado estaría conectado y podría beneficiarse de los apoyos, y las becas que proporciona la estructura olímpica para los alumnos y los jóvenes investigadores. " 55

${ }^{54}$ COE. (2007b). Informe sobre las actividades del Comité Olímpico Español mayo 2006 - mayo 2007. Madrid: COE.

${ }^{55}$ De entrevista mantenida con la Dra. M ${ }^{a}$ Eugenia Martínez Gorroño, en la sede del CEO de la UAM, en el Campus de Cantoblanco, (Madrid) el 14 de abril del 2015. 


\section{CONCLUSIONES} respuestas:

En relación a los objetivos planteados, hemos obtenido las siguientes

- Los Centros de Estudios Olímpicos son delegaciones de la Academia Olímpica Española dentro de la estructura de las Universidades españolas.

- El objetivo principal de estas instituciones es colaborar con la Academia Olímpica Española en la difusión del Movimiento Olímpico: historia, filosofía, valores e investigación.

- En relación al trabajo desarrollado, los CEOs han organizado actividades, seminarios, jornadas, etc. sobre temas Olímpicos, han llevado a cabo las Sesiones Anuales de la Academia Olímpica Española y, en algunos casos como es el del Centro de la Universidad Autónoma de Madrid, han sido impulsores de la múltiples publicaciones relacionadas con sus objetivos de creación, para la difusión del Movimiento Olímpico y sus circunstancias. Todas estas actividad

Consecuentemente, podemos concluir que:

El Olimpismo Moderno se inició en el contexto universitario. Esta es la circunstancia y el primero de los hechos históricos para argumentar la creación de los Centros de Estudios Olímpicos dentro de las Universidades. Esta consideración fue definitiva en la génesis de la orientación universitaria de las iniciativas promovidas, según afirma Conrado Durántez Corral, impulsor de la creación de las Academias Olímpicas Nacionales a través de la creación de la Academia Olímpica Española, pionera en este género. La capacidad y calidad de difusión del Movimiento Olímpico que puede proporcionar la comunidad universitaria han sido aspectos fundamentales para considerar estos centros como delegaciones de la AOE.

La celebración de múltiples actividades que han venido realizándose a través de estos centros, en estrecha colaboración con la AOE como: las Sesiones Anuales de la AOE, la selección de los estudiantes que representan a la AOE en cada edición de la Sesión para Jóvenes Participantes de la AOI, las publicaciones sobre la Historia Olímpica, las investigaciones y trabajos sobre la Educación y Valores Olímpicos que han venido realizándose a través de estos centros, dejan de manifiesto y materializan la contribución de los Centros de Estudios Olímpicos al Movimiento Olímpico, cumpliendo así los objetivos que justificaban su creación y sirviendo de ejemplo para otras Academias Nacionales que ya han imitado esta iniciativa, como 
la Academia Olímpica Argentina, contribuyendo así a una mayor expansión del Olimpismo.

\section{FUENTES Y BIBLIOGRAFÍA}

\subsection{Archivos}

- Archivo de la Academia Olímpica Española

- Archivo del Comité Olímpico Español

- Archivo del Centro de Estudios Olímpicos de la Universidad Autónoma de Madrid.

\subsection{Fuentes primarias}

\subsubsection{Fuentes orales}

- Conrado Durántez Corral. Presidente de la Academia Olímpica Española. Entrevista realizada en Madrid, en la Sede del Comité Olímpico Español, el 18 de Abril de 2013.

- Conrado Durántez Corral, Presidente de la Academia Olímpica Española. Entrevista realizada en Madrid, en la Sede del Comité Olímpico Español, el 7 de Mayo de 2013.

- $\quad \mathrm{M}^{\mathrm{a}}$ Eugenia Martínez Gorroño, Directora del CEO de la UAM y Miembro de la Academia Olímpica Española y Directora del Centro de Estudios Olímpicos de la Universidad Autónoma de Madrid.Entrevista realizada en la sede del CEO de la UAM, en el Campus de Cantoblanco, (Madrid) el 14 de abril del 2015.

\subsubsection{Fuentes de archivo}

- Actas de la Academia Olímpica Española XVI Sesión 1986. Madrid. Comité Olímpico Español.

- Convenio firmado entre el COE y la Universidad Autónoma de Madrid para la creación del CEO de la UAM.

- Convenios firmados entre el COE y otras Universidades del Estado Español.

Citius, Altius, Fortius Volumen 8 ne 2 Noviembre, 2015. ISNN. 2340-9886.

http://dx.doi.org/10.15366/eitius2015.8.2 
- Documentación procedente del archivo de los Centros de Estudios Olímpicos creados hasta la fecha, depositada en el Archivo de la AOE.

\subsection{Bibliografía}

- AOE. (1990). Academia Olímpica Española XXII Sesión: XX Aniversario. Madrid: Comité Olímpico Español.

- AOE. (2009). Academia Olímpica Española. 1968-2008. XL Aniversario. Madrid: COE.

- Bédarida, F. (1998). Definición, método y práctica de la Historia del Tiempo Presente. Cuadernos de Historia contemporánea, 20, 19-27.

- Best, J.M. (1982). Cómo investigar en educación. Ediciones Morata. Madrid.

- Canedo Ibarra, S. P. (2009). Contribución al estudio del aprendizaje de las ciencias experimentales en la educación infantil: cambio conceptual y construcción de modelos científicos precursores. Tesis doctoral en el Programa de doctorado en didáctica de las ciencias experimentales y la matemática. Universitat de Barcelona.

- COE. (2007a). ler Congreso Nacional de Centros de Estudios Olímpicos. Universidad de Granada, 22 y 23 de marzo de 2007. Madrid: COE.

- COE. (2007b). Informe sobre las actividades del Comité Olímpico Español mayo 2006 - mayo 2007. Madrid: COE.

- COE. (2007a). ler Congreso Nacional de Diplomados de Olimpia. MadridComité Olímpico Español 4 y 5 de octubre de 2008. Madrid: COE.

- Cuesta Bustillo, J. (1998). Memoria e historia. Un estado de la cuestión. AYER (32), 203-229.

- Durántez Corral, C. (1988). La Academia Olímpica Internacional. Madrid. Comité Olímpico Español.

- Durántez Corral, C. (2003). Academias Olímpicas Nacionales. Madrid. Comité Olímpico Español publicaciones Academia Olímpica Española. 
- Durántez Corral, C. (2008). Academia Olímpica Española 1968-2008 XL Aniversario. Madrid. Academia Olímpica Española. Madrid.

- Durántez Corral, C. (2012). El Comité Olímpico Español: Un siglo de historia. Citius, Altius Fortius. Humanismo, Sociedad y Deporte: Investigaciones y ensayos, 5(2)- 2012, 9-48. Centro de Estudios Olímpicos de la Universidad Autónoma de Madrid.

- El País. (2010). Juan Antonio Samaranch: su vida en EL PAIS.com. El País digital. Recuperado de http://www.elpais.com/especial/juan-antonio$\underline{\text { samaranch/vida.html }}$

- Fazio Vengoa, H. (1998). La historia del tiempo presente: una historia en construcción. Historia Crítica, 17, 47-57.

- Hammer, D. y Wildavsky, A. (1990). La entrevista semi-estructurada de final abierto. Aproximación a una guía operativa. Historia y Fuente Oral, 4, 23-61.

- Martínez Gorroño, M.E. (1990). Fuentes orales para una aproximación al exilio femenino en Colombia. Espacio, Tiempo y Forma. Serie V. Historia Contemporánea.

- Martínez Gorroño, M.E. (1996). Metodología y fuentes en el estudio del exilio español en Colombia a consecuencia de la guerra civil 1936-1939. En J.M Trujillano Sánchez y P. Díaz Sánchez (Ed.), Actas y Jornadas Historia y Fuentes orales-testimonios orales y escritos, (pp. 253-258). Ávila, España: Fundación Cultural Santa Teresa.

- Martínez Gorroño, M.E., Durántez Corral, C. (2011). Movimiento Olímpico: historia y retos actuales. Actas XLIV sesión oficial de la Academia Olímpica Española. 14-19 de noviembre del 2011. Centro de Estudios Olímpicos de la Universidad Autónoma de Madrid. Servicio de publicaciones de la UAM.

- Martínez Gorroño, M. E. (2015). Conrado Durántez Corral: La Academia Olímpica Española y el olimpismo. Materiales para la Historia del Deporte, [S.1.], 158-181. Universidad Pablo de Olavide de Sevilla. ISSN 1887-9586. Disponible en:

$<$ http://www.upo.es/revistas/index.php/materiales historia deporte/article/vie w/1230/985>. 
- Mateos López, A. (1998). Historia, Memoria, Tiempo Presente. Hispania Nova, (1). Recuperado de http://hispanianova.rediris.es/general/articulo/004/art004.htm.

- Mudrovcic, M. I. (2000). Algunas consideraciones epistemológicas para una "Historia del Presente". Hispania Nova 1998-2000. Recuperado de http://hispanianova.rediris.es/general/articulo/013/art013.htm

- Müller, N. (2004). Educación Olímpica. Lecciones universitarias olímpicas [artículo en línea]. Barcelona: Centre d’Estudis Olímpics (UAB). Cátedra Internacional de Olimpismo (CIO-UAB). Recuperado de http://olympicstudies.uab.es/cast/lectures/web/pdf/spa_muller.pdf

- Rodríguez Gómez, G., García Jiménez, E. (1996). Metodología de la investigación cualitativa. Granada: Ediciones Aljibe.

- Tarín Mariscal, M. (2013). Historia de la génesis de la Academia Olímpica Española. Contexto, herencia y consecuencias de su creación. Citius, Altius Fortius. Humanismo, Sociedad y Deporte: Investigaciones y ensayos Vol. $6 \mathrm{n}^{\mathrm{o}}$ 2, pp. 39-59. Centro de Estudios Olímpicos de la Universidad Autónoma de Madrid. http://cdeporte.rediris.es/revcaf/CitiusAltiusFortius.html

\subsection{Otras fuentes}

- Página oficial de la Academia Olímpica Española. http://www.coe.es/2012/HomeOlimpismo.nsf/FHomeOlimpismoN3?ReadFor m\&Nivel3=Academia\%20O1\%C3\%ADmpica

- Página oficial de la Academia Olímpica Internacional http://www.ioa.org.gr/index.php?lang=en 